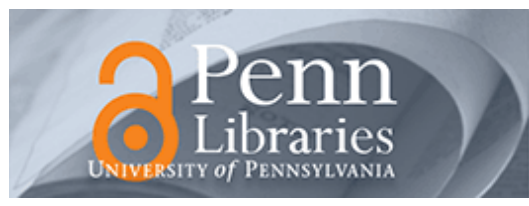

University of Pennsylvania
ScholarlyCommons

Departmental Papers (Obstetrics and Gynecology)

Department of Obstetrics and Gynecology

2013

\title{
An Exploratory, Randomized, Crossover MRI Study of Microbicide Delivery with the SILCS Diaphragm Compared to a Vaginal Applicator
}

Sara Pentlicky

University of Pennsylvania

Mark Rosen

University of Pennsylvania

Patricia S. Coffey

M. Kilbourne-Brook

A. Shaunik

University of Pennsylvania

See next page for additional authors

Follow this and additional works at: https://repository.upenn.edu/obgyn_papers

Part of the Medicine and Health Sciences Commons

\section{Recommended Citation}

Pentlicky, Sara; Rosen, Mark; Coffey, Patricia S.; Kilbourne-Brook, M.; Shaunik, A.; Schreiber, Courtney A.; and Barnhart, Kurt, "An Exploratory, Randomized, Crossover MRI Study of Microbicide Delivery with the SILCS Diaphragm Compared to a Vaginal Applicator" (2013). Departmental Papers (Obstetrics and Gynecology). 4.

https://repository.upenn.edu/obgyn_papers/4 


\title{
An Exploratory, Randomized, Crossover MRI Study of Microbicide Delivery with the SILCS Diaphragm Compared to a Vaginal Applicator
}

\begin{abstract}
Background-Microbicide gels studied for HIV prevention often are delivered via a single-use vaginal applicator. Using a contraceptive diaphragm such as the SILCS diaphragm for gel delivery could have advantages, including lower cost and additional pregnancy prevention.
\end{abstract}

Study Design - We performed an exploratory, nonblinded, randomized, crossover study among healthy, sexually active, nonpregnant women. Using BufferGel ${ }^{\oplus}$, we evaluated three microbicide delivery methods for gel distribution and retention: SILCS single-sided gel delivery, SILCS double-sided gel delivery and a vaginal applicator (without SILCS). Magnetic resonance images were taken at baseline, after gel insertion, and immediately and $6 \mathrm{~h}$ after simulated intercourse. Three women completed all gel delivery methods described in this article.

Results-Magnetic resonance imaging analysis indicated similar gel spread in the vagina among all three methods. SILCS single-sided gel application resulted in the most consistent longitudinal coverage; SILCS double-sided gel application was the most consistent in the transverse dimension.

Conclusions-Gel coverage was similar with all three methods. These results suggest that the SILCS microbicide delivery system is comparable to vaginal applicators for delivery of gel products intravaginally.

\section{Disciplines}

Medicine and Health Sciences

\section{Author(s)}

Sara Pentlicky, Mark Rosen, Patricia S. Coffey, M. Kilbourne-Brook, A. Shaunik, Courtney A. Schreiber, and Kurt Barnhart 


\title{
An exploratory, randomized, crossover MRI study of microbicide delivery with the SILCS diaphragm compared to a vaginal applicator
}

\author{
Sara Pentlicky ${ }^{\mathrm{a}}$, Mark Rosen ${ }^{\mathrm{a}}$, Patricia S. Coffey ${ }^{\mathrm{b}}$, M. Kilbourne-Brook ${ }^{\mathrm{b}}$, A. Shaunik $^{\mathrm{a}}$, \\ Courtney A. Schreiber ${ }^{a}$, and Kurt Barnhart ${ }^{\mathrm{a}}{ }^{*}$ \\ aUniversity of Pennsylvania, Philadelphia, PA 19104, USA \\ bProgram for Appropriate Technology in Health (PATH), Seattle, WA 98121, USA
}

\begin{abstract}
Background-Microbicide gels studied for HIV prevention often are delivered via a single-use vaginal applicator. Using a contraceptive diaphragm such as the SILCS diaphragm for gel delivery could have advantages, including lower cost and additional pregnancy prevention.
\end{abstract}

Study Design-We performed an exploratory, nonblinded, randomized, crossover study among healthy, sexually active, nonpregnant women. Using BufferGel®, we evaluated three microbicide delivery methods for gel distribution and retention: SILCS single-sided gel delivery, SILCS double-sided gel delivery and a vaginal applicator (without SILCS). Magnetic resonance images were taken at baseline, after gel insertion, and immediately and $6 \mathrm{~h}$ after simulated intercourse. Three women completed all gel delivery methods described in this article.

Results-Magnetic resonance imaging analysis indicated similar gel spread in the vagina among all three methods. SILCS single-sided gel application resulted in the most consistent longitudinal coverage; SILCS double-sided gel application was the most consistent in the transverse dimension.

Conclusions-Gel coverage was similar with all three methods. These results suggest that the SILCS microbicide delivery system is comparable to vaginal applicators for delivery of gel products intravaginally.

\section{Keywords}

SILCS diaphragm; Microbicide delivery; MRI; Vaginal applicator

\section{Introduction}

The past decade has fostered the development of microbicides intended to prevent HIV transmission. A recent microbicide trial demonstrated the ability of a vaginal agent to prevent HIV and herpes simplex virus type 2 transmission [1,2]. In most studies, the gel is delivered by a single-use, prefilled vaginal applicator which works well in a research environment, but may not be appropriate or cost-effective for long-term use of a microbicide gel $[3,4]$. Using a contraceptive diaphragm such as the SILCS diaphragm for gel delivery could have advantages, including lower cost and prevention of unintended pregnancy.

(C) 2013 Elsevier Inc. All rights reserved.

*Corresponding author. 3701 Markekt Street, Philadelphia, PA 19194. Tel.: +1 215662 2974; fax: +1 2153495512. kbarnhart@obgyn.upenn.edu (K. Barnhart). 
The SILCS diaphragm (SILCS), named for SILCS, Inc., an early partner in its development, is a reusable, single-sized contraceptive barrier that improves on features that limit use and acceptability of traditional diaphragms. Specifically, SILCS has a contoured spring that allows it to fit most women comfortably. SILCS acceptability and barrier effectiveness have been reported elsewhere [5-7]. SILCS is an investigational device not yet approved for use by any regulatory authority.

One vaginal microbicide currently being studied is an investigational drug, BufferGel ${ }^{\circledR}$ (BG). BG uses an acidic buffering action to maintain the mild, protective acidity of the vagina in the presence of semen. The safety of BG has been confirmed [8-10], and contraceptive efficacy compares well to nonoxynol-9 when used with a diaphragm [11].

Since many microbicide gel studies employ vaginal applicators, little is known about the impact of an alternative delivery system on gel distribution. A diaphragm could influence gel retention, distribution and user acceptability; however, if gel delivery from a diaphragm is acceptable, feasible and comparable to gel delivery from a vaginal applicator, a diaphragm delivery system could also improve women's options for dual protection. This study evaluated how gel delivery with SILCS compares to gel delivery from a traditional vaginal applicator.

\section{Materials and methods}

This was an exploratory, nonblinded, randomized, crossover study conducted at the Hospital of the University of Pennsylvania in Philadelphia, PA, between February 2009 and March 2010. The study was approved by the Institutional Review Board of the University of Pennsylvania and Program for Appropriate Technology in Health (PATH) Research Ethics Committee. We used magnetic resonance imaging (MRI) to evaluate gel distribution and retention.

Women, ages 18 to 45 years, who were sexually active with a male partner in the last year and not pregnant in the past 10 weeks, were screened and enrolled. All women reported regular menstrual cycles, were not breastfeeding, had no signs of infection or inflammation of the genitourinary tract, and had no contraindications to an MRI. Women who were using an intrauterine device or a vaginal ring for contraception were excluded. Before undergoing the MRI portion of the study, women were assessed for baseline diaphragm size and practiced inserting the SILCS. Study staff confirmed the correct placement.

The SILCS diaphragms used in this study were provided by PATH and manufactured by Molded Rubber Products Corporation (MRPC, Butler, WI). BG was provided by ReProtect, Inc. (Baltimore, MD). BG was mixed with a contrast agent, gadolinium (Omniscan ${ }^{\mathrm{TM}}$, GE Healthcare), to a dilution of 1:100 to allow visualization by MRI.

\subsection{Gel delivery methods and visits}

This article reports on microbicide gel delivered by three different gel methods: (A) SILCS with $5 \mathrm{~mL}$ of BG applied to the cervical side of the device, (B) SILCS with $2.5 \mathrm{~mL}$ of BG applied to the cervical side of the device and $2.5 \mathrm{~mL}$ inserted directly into the vagina after the diaphragm was placed and (C) $5 \mathrm{~mL}$ of BG inserted into the vagina with the vaginal applicator (without SILCS). Each participant was assigned a unique and random order in which the gel delivery methods were tested, which was determined by a randomly generated number provided by the Investigational Drug Service.

All study visits were conducted around the menstrual cycle. If the participant was sterilized or using hormonal contraception, she scheduled study visits at any time except during her 
menses. If the participant was not using hormonal contraception, she scheduled her visits between cycle days 6 through 12. The minimum time between each visit was 4 days since previous data demonstrated that a 48-h washout period was necessary to no longer detect gel on the MR images [12].

\subsection{MRI procedures}

At the first study visit, the baseline MRI was performed, which coincided with an interim history, a urine pregnancy test and assignment to one of the three gel delivery methods. The second (presimulation) MRI was performed within $15 \mathrm{~min}$ of gel insertion. Next, the participant was asked to simulate vaginal intercourse (30 thrusts) with a model phallus (Centaur 2, Northampton, MA) under a standard study protocol $[13,14]$. After completion, a third (postsimulation) MRI was performed. Then participants left the clinic and assumed normal daily activities. They were asked to refrain from vaginal intercourse, and those using SILCS were instructed to leave it in place until after the last MRI. After $6 \mathrm{~h}$, the participants returned for the fourth (delayed) MRI. After each MRI, the participants assessed the acceptability of the gel delivery system being used on that day. Each participant returned for her next visit no earlier than 4 days later to assess the next gel delivery method. Participants completed at least three study visits, one for each of the gel delivery methods.

\subsection{MRI technique}

All MRIs were performed on a 1.5-T system (Sonata, Siemens, Erlanger, Germany) equipped with anterior and posterior array coils. The MRI technique was similar to that previously described [13-15]. Baseline imaging included axial and sagittal T2-weighted imaging, as well as thin-slice volumetric T1-weighted imaging to exclude hemorrhagic contents in the vagina that could mimic gadolinium-doped gel. After gel/diaphragm insertion, sagittal T2- and T1-weighted imaging was repeated twice (before and after simulated intercourse). The sagittal imaging series was then repeated at $6 \mathrm{~h}$.

\subsection{Statistical analysis}

2.4.1. Sample size determination-In order to detect a $20 \%$ difference in surface coverage between any of the two groups with $80 \%$ power, given an alpha of 0.05 and a correlation coefficient of 0.7 , it was determined that we needed six subjects. Mean surface contact value and standard deviation were calculated using data from a previous study $[14,15]$. The crossover design allowed for efficient and accurate evaluation of the differences among methods since there are within-subject and between-subject comparisons. In this design, each of the participants acted as her own control.

\subsection{Anatomical measurements}

MR images were analyzed by a single, blinded observer who read and performed all specified measurements. All image analyses were performed using a three-dimensional (3D) workstation (TeraRecon®, Foster City, CA). Vaginal dimensions were assessed on preinsertion $\mathrm{T} 2$-weighted images from method $\mathrm{C}$ (vaginal applicator) and have been previously described $[13,15]$. The vaginal "linear length" was measured segmentally in the sagittal plane from the introitus to the posterior fornix. Transverse measurements were taken from the axial images at five anatomic locations: the posterior vaginal fornix, $1 \mathrm{~cm}$ below the anterior cervix, the vaginal flexure and $1 \mathrm{~cm}$ above the introitus. These measurements were added to create a single "summed" transverse dimension [13-15]. Baseline measurements are shown in Fig. 1.

During the presimulation, postsimulation and delayed MRIs, the sagittal and longitudinal measurements were repeated using the T1-weighted images to document gel spread. Oblique 
reformatted planes were created to present the maximal transverse spread at each anatomic location. The linear extent at any anatomic location was measured as the sum of the separate continuous linear regions.

Linear surface contact is the sum of the width of gel in the posterior fornix (anteriorposterior plane) and the transverse gel measurement at the four other demarcated anatomic sites. Linear spread from the posterior fornix to the introitus was assessed in the sagittal plane. The linear gel spread measurements (longitudinal and summed transverse) were divided by similar measurements of the vaginal extent on the preinsertion T2-weighted images to create percent longitudinal and transverse measurements of gel spread (i.e., percent surface contact).

\subsection{Statistical methods}

Comparisons of linear vaginal gel spread were made using Wilcoxon signed-rank tests since we could not predict that the data would be normally distributed. SAS (Cary, NC) software was used for statistical analysis. Analysis included $\chi^{2}$ tests, paired $t$ test and analysis of variance (ANOVA). Descriptive statistics were employed to provide preliminary data on acceptability.

\section{Results}

\subsection{Demographic characteristics}

Nine women were screened, and six enrolled in the study. They were on average 26 years old (range 23-32 years). Three of the six participants were Caucasian, two were African American, and one was Asian. Four of the six participants were using hormonal contraception during their participation, and all were nulliparous.

\subsection{Gel coverage assessment}

Initial MRI assessments from the six women in this study showed unexpected results when using the SILCS diaphragm. There were larger than expected differences between the SILCS methods A and B when compared to method C (Fig. 2). Based on qualitative data (not reported here), we surmised that this was due to difficulty with expressing gel from a sachet which resulted in inconsistent gel volumes introduced into the vagina. Since this was an exploratory study, we revised the gel packaging, and three women repeated methods A and B using the modified gel packaging. This additional step ensured that a uniform gel volume was applied to the SILCS and resulted in MRI scans with more comparable gel volume in the vagina. The differences between the original gel delivery methods $\mathrm{A}$ and $\mathrm{B}$ (identified as $\mathrm{A}_{\mathrm{o}}$ and $\mathrm{B}_{\mathrm{o}}$ ) and the new methods $\mathrm{A}$ and $\mathrm{B}$ with modified packaging (identified as $A_{n}$ and $B_{n}$ ) were statistically different. The difference was attributable to the poor functionality of the original gel packaging rather than the ability of the SILCS to deliver the gel once it was inserted vaginally. Since the gel spread seen with the revised gel packaging was more consistent with gel images from previous studies, we accepted the revised packaging as a more reliable method for delivering a consistent gel dose. All six participants completed 12 MRIs; the three women who repeated methods A and B, each with the updated gel packaging, underwent 8 additional MRIs for a total of 20 MRIs. Only results from the new methods $\left(A_{n}\right.$ and $\left.B_{n}\right)$ are presented here and will be referred to as methods $A$ and $\mathrm{B}$ from here forward.

Longitudinal and transverse gel coverage assessments for methods A, B and C were compared at the various study visits and are presented in Table 1 . When assessing changes in gel spread over time, there were four statistically significant differences in gel spread. First, in method A, as time passed, the transverse gel spread decreased. Second, in method 
B, as time passed, longitudinal gel spread increased. Lastly, in method C, in both the transverse and longitudinal directions, the gel spread increased with time. Comparisons for gel spread among the methods, at each specific time point, are presented in Fig. 3. No statistically significant differences between the absolute spread and percent spread were found when comparing methods A, B and C at each time point.

\subsection{Qualitative pattern of spread}

Upon review of 3D MRIs, there were qualitative differences in spread among methods of gel delivery. The pattern of gel spread using an applicator (Fig. 2-i) was similar to previous studies [13]. The gel forms an image of the vaginal contour including the projection of the cervix into the vaginal canal. The gel pattern seen with SILCS, however, conforms to the shape of the SILCS diaphragm, resulting in an outline of the device. Gel is clearly seen "inside" the dome of the SILCS and outlining its rim (Fig. 1-iii). Little gel was visible below the SILCS in method A or B.

\subsection{Adverse events}

There were no major safety concerns or serious adverse events in the study. One subject developed a yeast infection that was thought to be related to the study.

\section{Discussion}

This is the first randomized, prospective study that examines the use of the SILCS diaphragm as a gel delivery system. Our data demonstrate that there were no detectable differences between the gel delivery seen with the SILCS diaphragm and that seen with the vaginal applicator.

The design took advantage of paired comparison in the same women to enhance the ability to find objective differences in gel deployment among the methods. While it is a small sample, initial findings indicate that placing the entire volume of gel into the cervical cup prior to inserting the SILCS diaphragm (method A) provided the most consistent longitudinal coverage. Method B, where the gel volume was divided between the cervical side of the SILCS and the lower vagina, provided the most consistent transverse measurements. There were statistically significant differences in the transverse and longitudinal gel spread seen when a vaginal applicator was used to administer the gel in method C. In method C, transverse coverage increased with time, while the longitudinal coverage peaked after simulated intercourse. It is unclear if these variations are clinically relevant, and our results support the conclusion that this novel gel delivery system has similar capabilities as a vaginal applicator.

When comparing methods at each time point, we found that methods using the SILCS (methods A and B) resulted in higher longitudinal and transverse coverage at the pre- and postsimulation time points when compared to the vaginal applicator (method C). Since the transmission of sexually transmitted infections and the risk of pregnancy are highest during sexual intercourse, we are hopeful that the coverage at pre- and post-intercourse simulation with the SILCS will be relevant in clinical application.

One qualitative difference noted was the variation of BG spread on MRI among the three groups. The gel pattern seen when delivered with the SILCS is different than when it is delivered by a vaginal applicator. It appears that there is "pooling" of gel along the edge of the SILCS around the cervix. This observation calls into question whether linear methods are the best descriptor for gel distribution when using devices such as the SILCS.

Quantification of these observations may require development of new measurement models 
to judge the "thickness" and "intactness" of the gel "ring" along the edge of the SILCS, which in turn could represent a different level of protection.

This study is limited by its small sample size. Unexpected findings required additional data collection so that only three women (instead of six) completed all gel delivery scenarios reported here. Notwithstanding, these pilot data are crucial in designing future studies.

The clinical significance of the differences and trends noted in this study needs to be further elucidated in larger studies. To date, it is unknown if it is optimal to maximize gel deployment to the transverse, longitudinal or upper aspects of the vagina. However, data suggest that adequate coverage at the pre- and postsimulation time points are clinically important in the prevention of pregnancy and infection. Our data show that the use of the SILCS to deliver gel intravaginally is comparable to the vaginal applicator. It is possible that once an effective microbicide gel is optimized, its use with the SILCS diaphragm could maximize dual protection options for women.

\section{Acknowledgments}

We thank Dr. Thomas R. Moench of ReProtect, Inc., for providing BufferGel® used in this study. Support for this research is made possible by the generous support of the American people through the United States Agency for International Development (USAID) under the terms of the HealthTech IV Cooperative Agreement \#GPHA-00-01-00005-00. The contents are the responsibility of PATH and do not necessarily reflect the views of USAID or the US Government.

\section{References}

1. Abdool Karim Q, Abdool Karim SS, Frohlich JA, et al. Effectiveness and safety of tenofovir gel, an antiretroviral microbicide, for the prevention of HIV infection in women. Science. 2010; 329:116874. [PubMed: 20643915]

2. Andrei G, Lisco A, Vanpouille C, et al. Topical tenofovir, a microbicide effective against HIV, inhibits herpes simplex virus-2 replication. Cell Host Microbe. 2011; 10:379-89. [PubMed: 22018238]

3. Vail J, Cohen J, Kelly K. Improving topical microbicide applicators for use in resource-poor settings. Am J Public Health. 2004; 94:1089-92. [PubMed: 15226124]

4. Cohen J, Steele M, Ureña F, Beksinska M. Microbicide applicators: understanding design preferences among women in the Dominican Republic and South Africa. Sex Transm Dis. 2007; 34:15-9. [PubMed: 16691157]

5. Coffey P, Kilbourne-Brook M, Beksinska M, Thongkrajai E. Short-term acceptability of a singlesize diaphragm among couples in South Africa and Thailand. J Fam Plann Reprod Health Care. 2008; 34:233-6. [PubMed: 18854068]

6. Coffey P, Kilbourne-Brook M, Brache V, Cochon L. Comparative acceptability of the SILCS and Ortho ALL-FLEX diaphragms among couples in the Dominican Republic. Contraception. 2008; 78:418-23. [PubMed: 18929740]

7. Schwartz J, Ballagh S, Creinin M, et al. SILCS diaphragm: postcoital testing of a new single-size contraceptive device. Contraception. 2008; 78:237-44. [PubMed: 18692615]

8. Mayer KH, Peipert J, Fleming T, et al. Safety and tolerability of BufferGel, a novel vaginal microbicide, in women in the United States. Clin Infect Dis. 2001; 32:476-82. [PubMed: 11170957]

9. van de Wijgert J, Fullem A, Kelly C, et al. Phase 1 trial of the topical microbicide BufferGel: safety results from four international sites. J Acquir Immune Defic Syndr. 2001; 26:21-7. [PubMed: 11176265]

10. Williams DL, Newman DR, Ballagh MD, et al. Phase I safety trial of two vaginal microbicide gels (Acidform or BufferGel) used with a diaphragm compared to KY Jelly used with a diaphragm. Sex Transm Dis. 2007; 34:1-8. [PubMed: 16755272]

11. Barnhart KT, Rosenberg MJ, MacKay HT, et al. Contraceptive efficacy of a novel spermicidal microbicide used with a diaphragm. Obstet Gynecol. 2007; 110:577-86. [PubMed: 17766603] 
12. Barnhart KT, Pretorius ES, Timbers K, Shera D, Shabbout M, Malamud D. Distribution of a 3.5$\mathrm{mL}(1.0 \%) \mathrm{C} 31 \mathrm{G}$ vaginal gel using magnetic resonance imaging. Contraception. 2005; 71:35761. [PubMed: 15854637]

13. Barnhart KT, Pretorius ES, Timbers K, Shera D, Shabbout M, Malamud D. In vivo distribution of a vaginal gel: MRI evaluation of the effects of gel volume, time, and simulated intercourse. Contraception. 2004; 70:498-505. [PubMed: 15541413]

14. Pretorius ES, Timbers K, Malamud D, Barnhart K. Magnetic resonance imaging to determine the distribution of a vaginal gel: before, during, and after both simulated and real intercourse. Contraception. 2002; 66:443-51. [PubMed: 12499038]

15. Barnhart KT, Izquierdo A, Pretorius ES, Shera DM, Shabbout M, Shaunik A. Baseline dimensions of the human vagina. Hum Reprod. 2006; 21:1618-22. [PubMed: 16478763] 

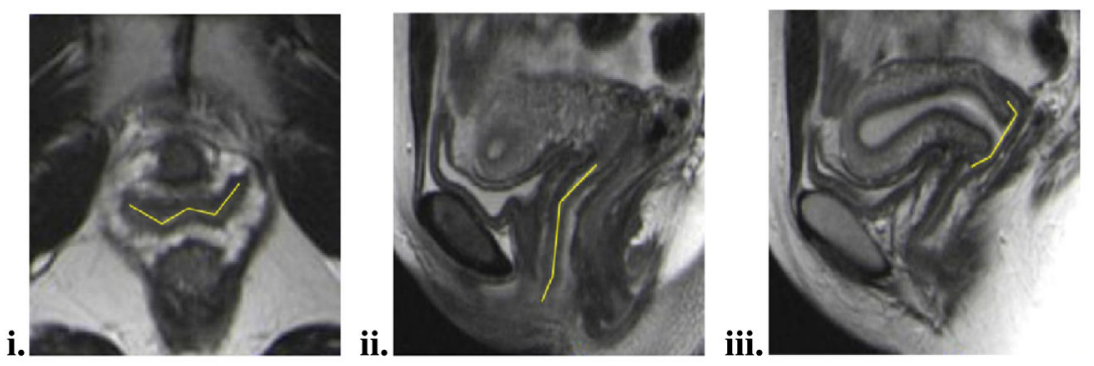

Fig. 1.

Example of linear vaginal measurements from T2-weighted MR image prior to gel insertion. (i) Axial image of the lower vagina ( $3 \mathrm{~cm}$ above introitus) with segmented linear tracing along vaginal mucosa. Transverse length at this location was $36 \mathrm{~mm}$. (ii) Sagittal T2weighted MR images demonstrating the longitudinal view and measurement of the vagina. Longitudinal vaginal length was $85 \mathrm{~mm}$. (iii) Sagittal image with linear tracing along vaginal mucosa around the cervix in an anterior-posterior fashion. 

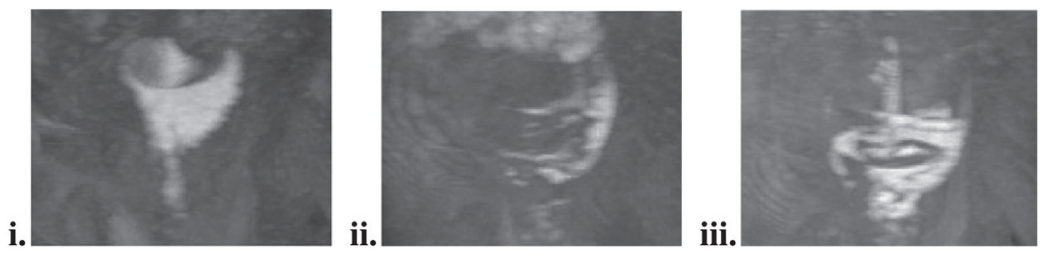

Fig. 2.

Visualization of the effect of gel volume administration. Maximum intensity projection images in the anterior-posterior view of the 3D T1-weighted MR images immediately after gel insertion for one subject. Gadolinium-doped gel is seen as bright areas against dark tissue background. (i) Gel distribution after applicator administration without SILCS device (method C). (ii) Gel distribution after split dosing of $2.5 \mathrm{~mL}$ each to the cervical and vaginal sides via method $\mathrm{B}_{\mathrm{o}}$. Note relative paucity of visualized gel (moderately bright areas in superior aspect of image represent bowel contents). (iii) Gel distribution after split dosing of $2.5 \mathrm{~mL}$ to the cervical side of the SILCS and the remaining $2.5 \mathrm{ml}$ inserted using an applicator (method $\left.B_{n}\right)$. 
i.

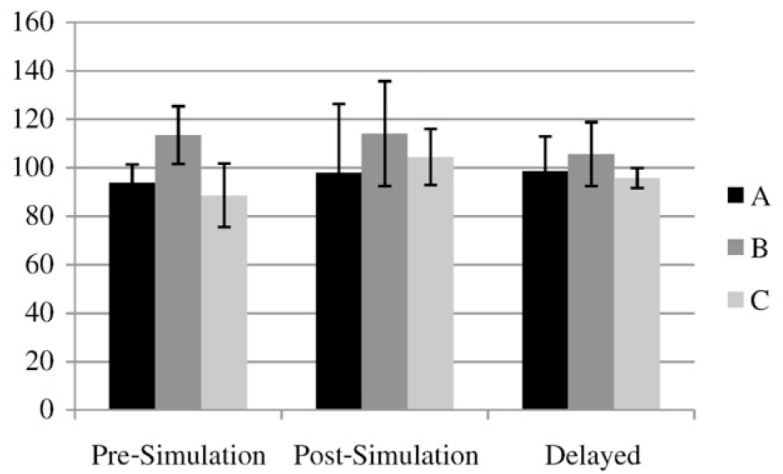

ii.

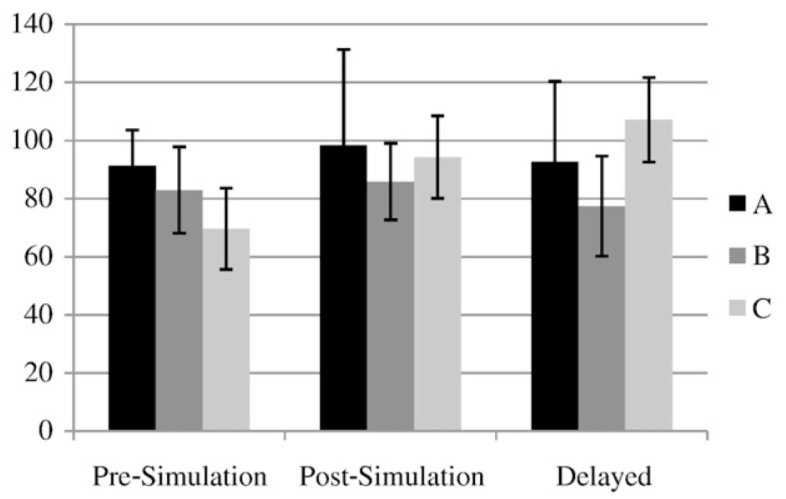

Fig. 3.

(i) Percentage longitudinal vaginal coverage at three time points. (ii) Percentage transverse vaginal coverage at three time points. The graphs are shown with error bars representing the standard deviation. Results may exceed $100 \%$ given postsimulation distention. 


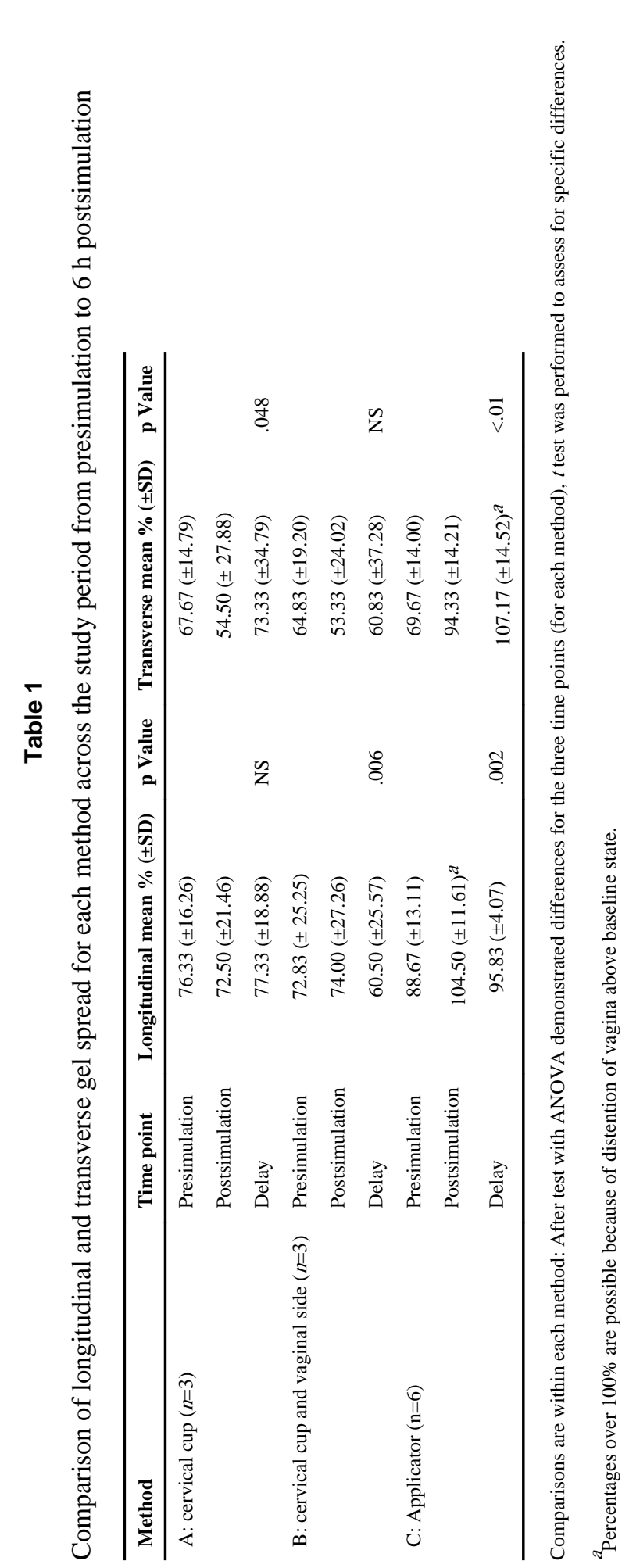

Contraception. Author manuscript; available in PMC 2014 February 01. 